\title{
Real-time MR-Thermometry and Dosimetry for interventional guidance on abdominal organs
}

\author{
Sébastien Roujol, M.Sc. ${ }^{1,2}$, Mario Ries, PhD $^{1}$, Bruno Quesson, PhD $^{1}$, Chrit Moonen, PhD $^{1}$, \\ Baudouin Denis de Senneville, $\mathbf{P h D}{ }^{1}$
}

1: Laboratory for Molecular and Functional Imaging: From Physiology to Therapy, UMR 5231 CNRS/Université Bordeaux 2, 146 rue Léo Saignat, F - 33076 Bordeaux, France

2: LaBRI, UMR 5800 CNRS/Université Bordeaux 1 - 351, cours de la Libération, F-33405 Talence, France

Address correspondence to:

Baudouin DENIS de SENNEVILLE

UMR 5231, Imagerie Moléculaire et Fonctionnelle

Université « Victor Segalen » Bordeaux 2

146, rue Léo Saignat, case 117

33076 Bordeaux, France

E-Mail: baudouin@imf.u-bordeaux2.fr

Tel: $\quad+33557574593$

Fax: $\quad+33557574597$

Acknowledgements: Ligue Nationale Contre le Cancer, Conseil Régional d'Aquitaine, Diagnostic Molecular Imaging EC-FP6-project LSHB-CT-2005-512146 "Agence National de Recherche (project MRgHIFU-ALKT)", Fondation InNaBioSanté (project ULTRAFIT)", Philips Medical System.

Word count: 4895 
ABTRACT

The use of Proton Resonance Frequency shift (PRF) based MR-Thermometry for interventional guidance on abdominal organs is hampered by the constant displacement of the target due to the respiratory cycle and the associated thermometry artifacts.

Ideally, a suitable MR-Thermometry method should for this role achieve a sub-second temporal

R1.17 resolution while maintaining a precision comparable to those achieved on static organs, while not $\mathrm{R} 2.1$ introducing significant processing latencies.

Here, a computationally effective processing pipeline for 2D image registration coupled with a R1.2 multi-baseline phase correction is proposed in conjunction with high frame-rate MRI as a possible solution.

The proposed MR-thermometry method was evaluated for five minutes at a frame-rate of 10

R1.1 images/s in the liver and the kidney of 11 healthy volunteers and achieved a precision of less than $2{ }^{\circ} \mathrm{C}$ in $70 \%$ of the pixels while delivering temperature and thermal dose maps on the fly. The ability to perform MR-Thermometry and Dosimetry in-vivo during a real intervention was demonstrated on a pig kidney during an HIFU-heating experiment.

\section{INTRODUCTION}

MR-thermometry relying on the water proton resonance frequency (PRF) is gaining importance for monitoring and guiding thermal therapies such as radio-frequency (1), laser (2) or focused $\mathrm{R} 2.2$ ultrasound thermal ablation (3,4,5). Typically, PRF-based MR-thermometry relies on the voxel-wise evaluation of phase differences between sequentially acquired gradient echo images.

However, for the use on abdominal organs, this renders the method very sensitive to motion artifacts and magnetic field changes. These motion artifacts can be coarsely classed into the two following types: Intra-scan motion artifacts and Inter-scan motion artifacts.

Intra-scan motion artifacts are caused by displacement during the MR-acquisition process and lead to image blurring and object ghosting. Commonly this type of artifact is addressed by the use of R1.1 fast MR-acquisition schemes. Alternatively, intra-scan motion artifacts can also be avoided by using respiratory gated sequences which, in turn, reduce the temporal resolution to the respiratory frequency.

Inter-scan motion artifacts are due to organ displacement between the MR-acquisitions. Although the image quality is not degraded, this leads to a mis-registration between subsequent phase images and thus to artifacts in the subtraction process. Furthermore, since any displacement or plastic deformation of the abdominal organs will in general also lead to a modified demagnetization field and 
thus to a change of the local magnetic field $(6,7,8)$, additional phase artifacts are introduced.

To overcome these problems, several correction strategies have been proposed, such as respiratory gating (9), cardiac triggering (10), navigator echoes (11), multi-baseline acquisition to sample periodic changes $(12,13)$ and reference-less phase corrections $(14)$.

Furthermore, although the local temperature is a precise indicator for the energy deposition, it does not directly allow to estimate tissue damage and thus to determine the therapy endpoint. For this purpose, the concept of the equivalent thermal dose was introduced to reflect the biological effects of elevated temperatures on tissue by Sapareto et al (15). The tissue destruction is achieved when the equivalent thermal dose exceeds the lethal dose (which is taken as $43{ }^{\circ} \mathrm{C}$ during $240 \mathrm{mn}$ ). Due to its exponential dependence on the temperature, artifacts in the temperature measurements have a large impact on the precision of the thermal dose estimate and will accumulate during the intervention due to the integration. Furthermore, since the temporal integration has to be performed on a voxel-by-voxel basis, a precise image registration is paramount for a precise calculation of the thermal dose.

Although the required correction schemes are computationally intensive, MR-image guidance of semi- or fully-automated interventions, such as high intensity focused ultrasound (HIFU) ablations, require the temperature and thermal dose measurements to be available in real-time with preferably short calculation latencies.

Here, a computationally efficient pipeline for 2D motion compensated PRF-thermometry and thermal dose measurements on moving abdominal organs is proposed. The method is designed to address both, inter-scan and intra-scan artifacts by applying high frame-rate MRI coupled with a realtime image registration of all incoming MR-images. Phase artifacts due to the periodical motion of the respiration cycle are addressed either by a multi-baseline strategy $(12,13)$, or alternatively by applying a phase correction based on a model of the phase variation in dependence of the principal displacement component which is adjusted from training-data on a voxel-by-voxel basis (16).

Furthermore, temporal filtering of the temperature maps allows to choose a balance between temporal resolution and the precision of the MR-thermometry which can be freely adjusted according to the employed interventional modality and the available signal-to-noise ratio of the target area.

All computational intensive calculations are off-loaded to a dedicated graphics processing unit (GPU) to allow sustained MR-thermometry and dosimetry under real-time conditions over durations of several minutes with short image latencies.

The potential of the real-time reconstruction pipeline to remove MR-thermometry artifacts dynamically is evaluated in-vivo on the abdomen of 11 healthy volunteers under free-breathing conditions, and on a pig kidney during an HIFU-heating experiment. 


\section{MATERIAL AND METHODS}

Figure 1 gives an overview of the employed data processing sequences for the multi-baseline and the linear phase modeling approaches.

Common to both approaches is the separation of the intervention into a pre-treatment step and a hyperthermia step. The pre-treatment step is performed prior to the intervention and records a reference dataset of magnitude and phase images during the motion cycle of the organ. In the multi-baseline approach this data is used to establish a lookup-table which serves as a reference phase during the hyperthermia step. For that purpose, the motion cycle must be sampled with a sufficient density in order to avoid discretization errors. With typical imaging frame-rates of 5-10 images per second and a respiration frequency of 3-5 s this pre-treatment step can be completed in a relatively short duration of $10-20$ s. For the linear phase-modeling approach the data collection of the pre-treatment step is subsequently used to fit a model function on a voxel-by-voxel basis in order to relate the recorded phase variations to their principal displacement component (PDC).

During the subsequent hyperthermia step, all incoming images are first co-registered to the common position of the reference image (17). Subsequently, the phase image is corrected for local magnetic field changes based on the data collected in the pre-treatment step and the temperature is calculated. Then, temporal filtering based on an infinite impulse-response (IIR) filter (18) is applied to the temperature maps to increase the Signal-To-Noise ratio (SNR) and finally the thermal dose is calculated.

The proposed approach takes benefit of a combined CPU/GPU architecture by offloading computational intensive calculations to the GPU and thus freeing the CPU for pipeline management and data preparation.

\section{Organ displacement estimation}

In the presented work a region based PDC estimation is initially performed on the $T_{2}{ }^{*}$ weighted images. This method serves also for preconditioning the more complex optical flow based image registration. Note that all corrections are estimated on the magnitude images but applied to the complex MR-images in order to avoid interpolation problems with spatial phase wraps.

PDC estimation: An affine transformation of six free parameters (two translations, two scales, a rotation and a shear) is estimated using a differential Gauss-Newton approach (19). Since the complexity of the estimated deformation is limited, the algorithm is unsuitable to cope with fold-over 
MR-artifacts, mixtures of static/dynamic parts of the entire field-of-view and complex motion patterns. Therefore it is restricted to a region of interest (ROI), which is manually set at the beginning of the intervention containing the full path of the targeted organ.

Optical flow based image registration: The implemented approach was originally proposed by Horn and Schunck (20) for optical image stabilization and computes a displacement field on a pixel-by-pixel basis. It includes a modification proposed by Cornelius and Kanade (21) which relaxes the intensity conservation condition from a global to a regional conservation. Finally, in order to optimize the computation time and to stabilize the convergence of the algorithm, a multi-resolution scheme was used (22) which iterates the registration algorithm from a four-fold down-sampled image step-by-step to the full image resolution.

\section{Susceptibility and displacement related phase changes with motion}

Although spatial displacements in phase images can be corrected by a suitable image registration, unwanted phase shifts generated by modified local magnetic susceptibility perturbations generally remain. This effect induces an apparent temperature modification which can bias or even completely mask the true temperature evolution induced by the energy deposition. Precise modeling of the inhomogeneous magnetic field in-vivo and under real-time conditions is difficult to achieve and thus several alternative simplified strategies have been proposed to allow to correct motion related errors in PRF-based MR thermometry $(11,12,13,14)$.

In the presented work, two correction strategies have been implemented, a multi-baseline approach and a linear phase model approach, which are both based on a training set composed of the first $N$ dynamic acquisitions in the time series (typically $N=100$ ), acquired prior to MR-thermometry.

Multi-baseline approach: The first $N$ dynamic acquisitions in the time series are stored in a look-up table $(12,13)$, which holds magnitude images and the corresponding registered phase images. During the intervention, arriving magnitude images are matched to the image with the closest correspondence in the look-up table by computing and comparing the inter-correlation coefficients. This task is performed on the CPU parallel to the image registration process performed on the GPU. Subsequently, the matching phase image of the GPU collection is subtracted to obtain a corrected temperature map.

Linear phase model approach: A second correction algorithm was implemented, which extends the multi-baseline approach by applying a model of local magnetic field changes to the phase data stored in 
the look-up table. The objective is to interpolate intermediate positions in order to reduce discretization errors and the required number of images in the correction data set, and to improve temperature accuracy by using a synthesized reference phase image. For this purpose, the first $N$ acquisitions are used to evaluate a linear relation between motion and phase variations. To prevent errors arising from the $2 \pi$-periodicity of the phase, all phase values $\varphi_{\mathrm{t}}(x, y)$ are first reordered over the time function of their corresponding displacement vector amplitude and subsequently phase unwrapped. Then, for each individual voxel, a system of $\mathrm{N}$ equations expressing the unwrapped registered phase $\left(\varphi_{\mathrm{t}}\right)$ as a linear

combination of the six free transformation parameters (noted $T_{i}^{t}, 0 \leq i<6$ is the index of the estimated transformation parameters, and $0 \leq t \leq N-1$ is the index of each image of the pre-treatment step) is given:

$$
\phi_{t}(x, y)=\sum_{i=0}^{5} T_{i}^{t} \cdot P_{i}(x, y)+P_{6}(x, y), \quad \forall \mathrm{t}, 0 \leq t \leq \mathrm{N}-1
$$

where $(x, y)$ denotes voxel coordinates, $\mathrm{P}_{\mathrm{i}},(0 \leq \mathrm{i}<6)$ are the unknowns and can be intuitively seen as a parameterized magnetic field model. This results in an overdetermined system resolved using a Singular Value Decomposition (SVD) .

For each image acquired after the learning phase, the transformation coefficients $T_{i}^{t}$ (calculated from the magnitude image registration) are used with the $P_{\mathrm{i}}$ (calculated from [1]) to compute a synthetic reference phase map $\left(\varphi_{\text {reco }}\right)$ as follows:

$$
\phi_{\text {reco }}(x, y)=\sum_{i=0}^{5} T_{i}^{t} \cdot P_{i}(x, y)+P_{6}(x, y)
$$

This reference phase map is then subtracted from the acquired motion corrected phase image to suppress the background phase prior to the temperature calculation.

\section{Correcting for local temperature aliasing}

Since the $2 \pi$ periodicity of the image phase can lead to aliasing artifacts in the temperature maps, a temporal phase unwrapping on a pixel-by-pixel basis is applied.

This process is valid under the condition that the temperature variation between two successive acquisitions does not create a phase variation greater than $\pi$. Note that on mobile targets, each phase 
image is registered to a common reference position and subsequently phase corrected, before temporal unwrapping is performed. Therefore, background-phase changes do not contribute to this limitation.

\section{Correcting for magnetic field drift}

Although recent MRI designs have excellent spatial magnetic field homogeneity, their field is not entirely stable over time when sustained high frame-rate imaging is applied (23). Therefore, the subtraction of phase images acquired at different times can be affected with a different bias for each

R1.7

$\mathrm{R} 2.10$ pixel $(8,24)$. In this study, this perturbation is corrected by subtracting a global temperature offset (a magnitude-weighted average is used to give less importance in poor signal area) obtained from a region of interest which is chosen in the moving organ, adjacent to the ablation area.

\section{Temporal temperature filtering}

An infinite impulse-response (IIR) low-pass filter of $5^{\text {th }}$ order (25), using an elliptic approximation in the denominator of the transfer function (also referred to as a Cauer filter), was used for temporal low-pass filtering of the calculated temperature values. The filter coefficients were designed using the signal processing toolbox of MATLAB with the following characteristics: $f_{\text {pass- }}$ band $=1.5 \mathrm{~Hz}$ to $8 \mathrm{~Hz}, \mathrm{f}_{\text {stop-band }}=4.5 \mathrm{~Hz}$ to $8.5 \mathrm{~Hz}$, ripple pass-band: $3 \mathrm{~dB}$, ripple stop-band: $50 \mathrm{~dB}$. Passband and stop-band were adjusted depending on the dynamic scan-time of the employed sequence to result in an overall temporal resolution of $2 \mathrm{~Hz}$.

\section{Experimental setup}

MRI imaging: Dynamic MR temperature imaging was performed on a Philips Achieva $1.5 \mathrm{~T}$ (Philips Healthcare, Best, The Netherlands) with a single-shot gradient recalled echo-planar (EPI) sequence (26). MR images were obtained using an in house developed reconstructor.

HIFU System: A MR compatible Philips HIFU prototype (Philips Healthcare, Helskinki, Finland) composed of a table top containing a 256 elements HIFU transducer (Imasonic, Besançon), integrated in the $1.5 \mathrm{~T}$ Achieva-Intera MRI was used to perform a temperature elevation. The transducer radius and aperture were $120 \mathrm{~mm}$ and $126 \mathrm{~mm}$, respectively, creating an ellipsoid focal point $\left(1 \times 1 \times 7 \mathrm{~mm}^{3}\right)$.

Reconstructor description: The reconstructor was a dual processor (INTEL $3.1 \mathrm{GHz}$ Penryn, four 
cores) workstation with $8 \mathrm{~GB}$ of RAM and dual $1 \mathrm{~GB} / \mathrm{s}$ network interface cards. The GPU was a NVIDIA GTX280 card with 1 GB of DRAM connected over a PCIe x16 link. The GPU implementation was realized using CUDA (27).

On-line in-vivo study: Dynamic MRI was performed under free-breathing conditions on the abdomen of 11 healthy volunteers under real-time conditions. An imaging frame-rate of 10 images/s was main-

$\mathrm{R} 1.1$

R1.10

$\mathrm{R} 1.16$ tained for 300 seconds of MR-imaging while MR-Thermometry was performed in real-time. The MR sequence employed the following parameters: 3000 dynamic sagittal images, one slice, $T R=100 \mathrm{~ms}$, $\mathrm{TE}=26 \mathrm{~ms}$, bandwidth in readout direction $=2085 \mathrm{~Hz}$, flip angle $=35^{\circ}, \mathrm{FOV}=256 \times 168 \mathrm{~mm}^{2}$, slice thickness $=6 \mathrm{~mm}$, matrix $=128 \times 84$, using a four element phased array body coil. Statistical evaluation of the temperature stability was performed on the kidney and the liver of each volunteer individually by averaging the temporal temperature standard deviation over a ROI which was manually set in an area with maximal SNR and avoiding areas showing a complex susceptibility distribution (such as organ boundaries or major vessels).

On-line in-vivo heating study on a pig kidney: MRI guided HIFU was performed in vivo in the kidney of a pig under general anesthesia. No focal point position adjustment with respect to target displacements was performed. The MR sequence employed the following parameters: 1500 dynamic sagittal

R1.1

$\mathrm{R} 1.10$

R1.16 images, one slice, $T R=100 \mathrm{~ms}, \mathrm{TE}=41 \mathrm{~ms}$, bandwidth in readout direction $=2085 \mathrm{~Hz}$, flip angle $=35^{\circ}$, FOV $=320 \times 140 \mathrm{~mm}^{2}$, slice thickness $=6 \mathrm{~mm}$, matrix $=128 \times 56$, using the integrated phased array coil of the HIFU system. The data processing sequence designed for the linear phase modelling approach was used to perform the MR-thermometry and dosimetry in real-time.

\section{RESULTS}

\section{Performance comparison between the CPU only and the CPU/GPU implementation}

Figure 1 details the computation time of each processing step of the two proposed pipelines for an image resolution of $128 \times 128$ pixels between the CPU only and the CPU/GPU implementation. The most time consuming task, the image registration, was accelerated by a factor of 3 for the PDC estimation and a factor of 10 for the optical flow computation. The resulting overall latency for the

R1.15 entire pipeline (including $13 \mathrm{~ms}$ for data transport and $1.2 \mathrm{~ms}$ for image reconstruction) was reduced from $95 \mathrm{~ms}$ (CPU only) to $27.3 \mathrm{~ms}$ (CPU/GPU).

Figure 2 details the precision improvement of each separate phase correction step for kidney 
and liver. Over the 11 human volunteers, the SNR was evaluated to $9.64 \pm 2.4(\min =7.2, \max =14.3)$ in the kidney and $7.5 \pm 3.1(\min =4.5, \max =14.3)$ in the liver. In average over all volunteers, the temperature stability is improved from an initial value of over $8{ }^{\circ} \mathrm{C}$ to $2.12{ }^{\circ} \mathrm{C}$ (kidney) and $2.66{ }^{\circ} \mathrm{C}$ (liver) using the multi-baseline approach, and to $1.5^{\circ} \mathrm{C}$ (kidney) and $2.16^{\circ} \mathrm{C}$ (liver), when the linear phase model is used. This precision can be furthermore improved by over $20 \%$ if a drift correction is applied: While a temperature standard deviation of $1.51^{\circ} \mathrm{C}$ (kidney) and $2.07{ }^{\circ} \mathrm{C}$ (liver) were obtained with the multi-baseline correction, the correction based on a linear phase model achieves a further reduction to $1.26{ }^{\circ} \mathrm{C}$ (kidney) and $1.77{ }^{\circ} \mathrm{C}$ (liver). Additional temporal filtering results in a final precision of $0.86{ }^{\circ} \mathrm{C}$ (multi-baseline), $0.79{ }^{\circ} \mathrm{C}$ (linear model) in the kidney and $1.05{ }^{\circ} \mathrm{C}, 0.98{ }^{\circ} \mathrm{C}$ in the liver. None of the data were rejected due to miss-registration or faulty phase correction due to the absence of spontaneous motion. Figure 3 shows as an example the least precise result of the examined volunteer group. Even in this case, both correction strategies ensured $2{ }^{\circ} \mathrm{C}$ of temperature stability in 70 $\%$ of all pixels of both the kidney and the liver.

\section{On-line in-vivo heating study on a pig kidney}

The overall temperature accuracy over the whole kidney was found to be $0.65{ }^{\circ} \mathrm{C} \pm 0.11$ (min=0.4, $\max =0.99$ ) with a measured SNR of 6 (although a SNR of 6 provides a theoritical

\section{R1.11}

$\mathrm{R} 1.12$ temperature standard deviation of $1.47^{\circ} \mathrm{C}$, the temporal filtering reduces the initial temporal resolution of $10 \mathrm{~Hz}$ down to $2 \mathrm{~Hz}$ improving the temperature precision by a factor of $\sqrt{5}$ ). Figure 4 .a shows the temperature image after 39 seconds of sonication corrected with the linear phase model approach. The evolution of the temperature at the focal point position is reported on figure 4.b. An hyperthermia of 12 ${ }^{\circ} \mathrm{C}$ was reached, which leads to a final thermal dose of $10 \%$ of the lethal dose as shown in figure 4.c (28).

\section{DISCUSSION}

\section{Organ displacement estimation:}

The main limitation of the presented approach is that through-plane motion can not be compensated, which places severe constrains on the type of motion that can be adressed and the $\mathrm{R} 2.4$ possible slice placement. For the case of respiratory induced motion, which predominantly causes a linear displacement of the abdominal organs in head-foot direction and to a lesser extend in anteriorposterior direction, this limits the choice of the slice placement to the coronal or sagital orientation. 
Although a full 3D motion compensation would be preferable, this is in practise hard to achieve with image based compensation techniques due to the inherent compromise between acquisition time and volume coverage.

PDC estimation: This approach was found to be well suited for targets which show only little plastic deformation during the respiratory cycle, such as the kidney. However, since such targets are in general in the vicinity of either elastic soft-tissue, such as digestive tubes or liver, or static tissue, such as the quadratus lumborum muscle or the vertebral column, the algorithm needs to be confined to a ROI encompassing only the full path of the selected target and excluding such problematic areas. For targets which display a complex spatially variant deformation such as the liver, this approach was found to be R2.5 unsuitable since a global adaption of only six free transformation parameters lead to a poor representation of the complex deformation. The main advantages of this approach are the short computation times and its robustness against noise and local intensity variations.

Optical flow based image registration: This technique allows to estimate complex organ deformations with sub-pixel precision on a voxel-by-voxel basis. Furthermore, the underlaying regularity constraint (20) which assumes a continuous differentiable motion field was found well suited for elastic targets which display local deformations during the respiratory cycle such as the liver or the spleen. Compared to Ross et al. (29), the implemented approach does not require any landmark identification and thus the estimated motion field is independent of preceding user interactions or preparational segmentation steps.

Although this approach is computationally intensive and thus challenging to implement for realtime imaging, the proposed CPU/GPU implementation renders this method compatible with sustained high frame-rate real-time imaging.

The main disadvantage of the original approach, as suggested by Horn and Schunk (19), is that it relies on the preservation of the global signal amplitude within the image. This renders the method sensible to contrast variations and to the effect of residual through-plane motion since any temporal change in the global intensity of the image is attributed to motion. The stability of the algorithm can be greatly improved by relaxing the global regularity constraint as suggested by Cornelius and Kanade (21). Although this modification limits mis-registration of the area of interest caused by contrast R2.10 changes or tissue disappearance outside the target region, this condition can still be violated. During hyperthermia several MR relevant tissue properties such as $T_{1}$ and $T_{2}$ relaxation times can change during heating process and thus lead to local signal intensity variations in the heated region. The impact of this miss-registration effect on large ablation areas, which may be used for advanced ablation 
strategies such as spatial volumetric temperature feedback (30), needs to be carefully investigated in future studies.

\section{Susceptibility related phase changes with motion:}

The proposed multi-baseline correction for susceptibility related phase changes is designed to correct MR-thermometry artifacts on abdominal organs which are subject to a periodic displacement due to the respiratory cycle. Since phase variations are "learned" in a preceding learning step and subsequently applied to correct the MR-thermometry during the intervention, the method can intrinsically not correct for MR-thermometry artifacts associated with spontaneous motion. If during hyperthermia new positions are observed, a recalibration of the phase correction data is required, which is a disadvantage compared to other correction approaches such as reference-less correction approaches (14). However, in practise this limitation is less severe, since the proposed correction approach is paired with to five respiratory cycles.

Furthermore, compared to reference-less correction methods (14), the proposed multi-baseline correction allows an accurate correction of susceptibility related phase changes even in regions with complex susceptibility distributions or signal discontinuities such as organ boundaries, since it requires neither a uniform susceptibility distribution in the target nor time-consuming 2D spatial phase-unwrapping steps (31).

In addition, since all images are co-registered to a common reference position before the phase corrections are applied, temporal aliasing of the temperature arising from the $2 \pi$ periodicity of the phase can simply be removed by applying a temporal unwrapping step on a voxel-by-voxel basis.

The proposed multi-baseline correction can be improved further by applying a linear phase model on a voxel-by-voxel basis with respect to the following three aspects: First, the correction is not constrained to positions present in the collection, but can also be interpolated to intermediate positions, which significantly reduces discretization errors and the required number of images in the correction data set (16). Second, while the multi-baseline based correction can intrinsically not correct for motion amplitudes higher than the ones observed in the learning phase, the phase model allows extrapolation and can still provide an estimate of the reference phase. Finally, since the reduced set of six $P_{i}$ coefficients is derived from the resolution of an overdetermined system of 50-100 reference images, noise

R1.3 will be reduced on the resynthesized_phase images. Ideally, the noise contribution on temperature uncertainty can thus be reduced by a factor $\sqrt{2}$ (as optimally $\sigma\left(\varphi_{\text {reco }}\right)=0$ ). Since the lower precision boundary of PRF-based MR-thermometry on static targets is directly proportional to the inverse of the SNR 
of the employed sequence (32), it is possible to evaluate the effectiveness of the correction strategies for dynamic phase artifacts by a direct comparison (assuming a noise free reference phase): On the 11 human volunteers the SNR study leads to a minimal achievable temperature standard deviation of 1.12 ${ }^{\circ} \mathrm{C} \pm 0.26$ (kidney, $\min =0.71, \max =1.48$ ) and $1.52{ }^{\circ} \mathrm{C} \pm 0.51$ (liver, $\min =0.71, \max =2.22$ ). The experimental study approaches those theoretical values when the linear phase model is used: After drift correction, the temperature precision in the kidney and the liver averaged over all volunteers were found to be $12 \%$ and $16 \%$ larger than the boundary imposed by the SNR, respectively (see figure 2 ).

On the other hand, the correction using the linear phase model introduces several new problems compared to the multi-baseline approach: The magnetic field perturbation is estimated assuming a simple linear magnetic field variation with organ displacement. Although this assumption holds in general for small displacements, the precision of this simple model must be carefully evaluated in regions displaying large motion amplitudes or large susceptibility variations, such as in the vicinity of the digestive tubes (see arrow (1) in figure 3.c), of the quadratus lumborum muscle (see arrow (2) in figure 3.c), of the vertebral column (see arrow (3) in figure 3.c), and in the upper part of the liver (see arrow (4) in figure 3.c). This can in practice be achieved by simply mapping the fitting error $\varepsilon(x, y)$ from equation [1]. This also allows to discard regions where low signal levels in conjunction with large susceptibility variations hampered a successful temporal phase unwrapping which is required to adjust the linear model to the phase data.

\section{Rapid acquisition with subsequent temporal filtering:}

The presented MR-Thermometry approach addresses intra-scan motion artifacts by applying high frame-rate MRI and the remaining inter-scan artifacts using image registration and a multibaseline phase correction. For the case of respiratory induced motion of abdominal organs, MR-acquisition times of typically $100 \mathrm{~ms}$ per slice or less are required to avoid intra-scan artifacts. This places severe restriction on the available processing time for image reconstruction, image registration and all MR-thermometry related calculations in order to maintain sustained real-time MR-thermometry.

Figure 1 shows that the motion estimation step remains the most time consuming step. For motion estimation based on/combined with optical flow, computation times can easily exceed typical MRacquisition times when the single CPU based implementation is used. The GPU based implementation allows to reduce the bulk computation time for all image processing steps below the used MR-acquisition time. This means that the achievable MR-thermometry frame-rate is below 30 images/s only limited by the constraints of the MR-sequence in order to satisfy the real-time condition. For interventional guidance such as energy deposition control or retroactive adjustment of the beam position, short laten- 
cies have a great importance. Denis de Senneville (13) showed that large image latencies have to be compensated with a temporal prediction and that the robustness of such algorithms improves with short latency. The two proposed corrections ensure a latency of $27.3 \mathrm{~ms}$ (image matrix of $128 \times 128$ ), which is suitable for interventional MR-guidance and is realizable on affordable commodity hardware.

Commonly, high frame-rate imaging for MR-thermometry is considered to be limited by the precision penalty imposed by the associated low SNRs on the temperature measurements. Since all MR temperature images in the time series are registered to the same reference position, temporal filtering can be applied to improve temperature accuracy prior to thermal dose calculation. This, in turn, allows to choose a balance between temporal resolution and the precision of the MR-thermometry which can be freely adjusted according to the employed interventional modality and the available SNR of the target area. For a direct tissue ablation using high temperature elevations of up to $30^{\circ} \mathrm{C}$, the temporal resolution will be privileged. On the other hand, recent experiments employing hyperthermia to control local drug delivery or gene expression (33), which represent a great potential for cancer therapy in kidney and liver, have much higher demands on the precision of the thermometry since it employs low temperature elevations of $4-5^{\circ} \mathrm{C}$. Theoretically, a perfect co-registration allows to double the SNR, and thus the precision of the temperature measurements, by reducing the bandwidth by a factor of 4 . In the volunteer study, a filter with a transition band between 0.15 and 0.45 resulted in an improvement of the precision of MR-thermometry by a factor 1.85 which corresponds well with the expected theoretical result.

\section{CONCLUSION}

The proposed approach for 2D motion compensated MR-thermometry and dosimetry addresses both, inter-scan and intra-scan motion artifacts, by applying high-framerate MRI coupled with real-time image registration and multi-baseline phase correction of all incoming MR-images. This, in conjunction with the use of parallel processing on affordable commodity graphics hardware, allows to achieve a sub-second temporal resolution with very short image latencies over sustained imaging periods of several minutes which is purely limited by the constraints of the MR-acquisition.

The temperature artifacts related to the periodic respiratory motion of the abdominal organs were found to be reduced to the boundary imposed by the SNR of the employed sequence in both, kidney and liver. Additional temporal filtering of the temperature maps allows to freely readjust the balance between temporal resolution and additional precision of MR-Thermometry.

The method was found robust and artifact free in all examined cases and well able to follow the temperature evolution of an in-vivo HIFU ablation. This renders the method well suitable for the MR- 
guidance of hyperthermia ablations in abdominal organs under free-breathing conditions and as the basis for more advanced automatic spatial and temporal temperature control algorithms used in conjunction with dynamic ultrasound beam-steering.

\section{REFERENCES}

(1) Laumonier H., Blanc J. F., Quesson B., Seror O., Laurent C., Bioulac-Sage P., Balabaud C., Trillaud H., Real-time monitoring of hepatocellular carcinoma radiofrequency ablation by quantitative temperature MRI, Semin Liver Dis, 2006; 26: 391-397.

(2) Puls R., Langner S., Rosenberg C., Hegenscheid K., Kuehn J.P., Noeckler K., Hosten N., Laser ablation of liver metastases from colorectal cancer with MR thermometry: 5-year survival., J Vasc Interv Radiol. 2009 Feb;20(2):225-34.

(3) Fry F. J., Precision high intensity focusing ultrasonic machines for surgery. Am. J. Phys. Med. 1958; 37, 152-156.

(4) Hindley J., Gedroyc W.M., Regan L., Stewart E., Tempany C., Hynyen K., Mcdannold N., Inbar Y., Itzchak Y., Rabinovici J., Kim HS., Geschwind J.F., Hesley G., Gostout B., Ehrenstein T., Hengst S., Sklair-Levy M., Shushan A., Jolesz F., MRI guidance of focused ultrasound therapy of uterine fibroids: early results., AJR Am J Roentgenol. 2004 Dec;183(6):1713-9.

(5) Hynynen K., McDannold N., Clement G., Jolesz F. A., Zadicario E., Killiany R., Moore T., and Rosen D., Preclinical testing of a phased array ultrasound system for MRI-guided noninvasive surgery of the brain-A primate study. Eur.J.Radiol., 2006; 59(2):149-56.

(6) De Poorter J., De Wagter C., De Deene Y., Thomson C., Stahlberg F., Achten E., The proton resonance frequency shift method compared with molecular diffusion for quantitative measurement of two dimensional time dependent temperature distribution in phantom, Journal of Magnetic Resonance Imaging, 1994, 103: 234-241.

(7) Young I. R., Hajnal J. V., Roberts I. G., Ling J. X., Hill-Cottingham R. J., Oatridge A., Wilson J. A., An evaluation of the effects of susceptibility changes on the water chemical shift method of temperature measurement in human peripheral muscle, Magnetic Resonance in Medicine, 1996, 
(8) Peters R. D., Henkelman R. M., Proton-resonance frequency shift MR thermometry is affected by changes in the electrical conductivity of tissue, Magnetic Resonance in Medicine, 2000, 43:62-71.

(9) Weidensteiner C., Kerioui N., Quesson B., de Senneville B. D., Trillaud H., Moonen C. T., Stability of real-time MR temperature mapping in healthy and diseased human liver, Journal of Magnetic Resonance Imaging, 2004, 19(4):438-46.

(10) Quesson B., Denis de Senneville B., Herigault G., Jaïs P., Moonen C. T. W., Dynamic cardiac MR temperature imaging with the Proton Resonance Frequency technique: a study on the feasibility and accuracy, ISMRM 2005, Floride.

(11) de Zwart J. A., Vimeux F. C., Palussière J., Salomir R., Quesson B., Delalande C., Moonen C. T. W., Online correction and visualization of motion during MRI-controlled hyperthermia, Magnetic Resonance in Medicine, 2001;45(1):128-37.

(12) Vigen K. K., Daniel B. L., Pauly J. M., Butts K., Triggered, navigated, multi-baseline method for proton resonance frequency temperature mapping with respiratory motion, Magnetic Resonance in Medicine, 2003, 50(5):1003-10.

(13) Denis de Senneville B., Mougenot C., Real time adaptive methods for treatment of mobile organs by MRI controlled High Intensity Focused Ultrasound, Magnetic Resonance in Medicine, 2007 Feb, 57(2):319-30.

(14) Rieke V., Vigen K. K., Sommer G., Daniel B. L., Pauly J. M., Butts K., Referenceless PRF shift thermometry, Magn Reson Med 2004;51(6):1223-31.

(15) Sapareto S. A., Dewey W. C. L., Thermal dose determination in cancer therapy, Int. J. Radiation Oncology Biol. Phys., 1984, 10:787-800.

(16) Hey S, Maclair G, de Senneville BD, Lepetit-Coiffe M, Berber Y, Köhler MO, Quesson B, Moonen CT, Ries M. Online correction of respiratory-induced field disturbances for continuous MRthermometry in the breast, Magn Reson Med. 2009 Jun;61(6):1494-9. 
(17) Maintz J. B. A., Viergever M. A., A survey of medical image registration, Medical Image Analysis, 1998, vol. 2, number 1, pp 1-36.

(18) Regalia, Adaptive IIR (infinite impulse response) filtering in signal processing and control (Electrical engineering and electronics series/90), Lavoisier, 1995

(19) Friston K. J., Ashburner J., Frith C. D., Poline J. B., Heather J. D. and Frackowiak R. S. J., Spatial registration and normalisation of images, Human Brain Mapping, 1995, 2:165-189.

(20) Schunck B.G. Horn K.P. - Determining optical flow. Artificial intelligence 1981; 17:pp. 185-203.

(21) Cornelius N., Kanade T., Adapting optical flow to measure object motion in reflectance and X-Ray image sequences, ACM SIGGRAPH/SIGART Interdisciplinary Workshop on motion: representation and perception, Toronto, Canada, April 83.

(22) Pratikakis I., Barillot C., Hellier P., Mémin E., Robust multiscale deformable registration of 3D ultrasound images. International Journal of Image and Graphics, 3(4):547-566, 2003.

(23) El-Sharkawy A. M., Schar M., Bottomley P. A., Atalar E., Monitoring and correcting spatiotemporal variations of the MR scanner's static magnetic field, MAGMA, 2006, 19(5):223-36.

(24) Riederer S. J., Recent technical advances in MR imaging of the abdomen, Journal of Magnetic Resonance Imaging, 1996, 6:822-832.

(25) Mitra S. K., Digital Signal Processing, second edition, McGraw-Hill, 2001.

(26) Bruder H., Fisher H., Reinfelder H.E., Schmitt F., Image reconstruction for EPI with nonequidistant k-space sampling, Magnetic Resonance in Medicine, 1992;23:311-323.

(27) NVIDIA Corporation, NVIDIA CUDA: Compute Unified Device Architecture, Programming Guide, 2.0 ed 2008, pp. 1-107.

(28) Seror O., Lepetit-Coiffé M., Quesson B., Trillaud H., Moonen C. T. W., Quantitative magnetic 
resonance temperature mapping for real-time monitoring of radiofrequency ablation of the liver: an ex vivo study, Eur Radiol. 2006 Oct;16(10):2265-74. Epub 2006 Apr 11.

(29) Ross J. C., Tranquebar R., Shanbhag D., Real-time liver motion compensation for MrgFUS, Proceedings of the 11th International Conference on Medical Image Computing and ComputerAssisted Intervention, 2008;11(Pt 2):806-813.

(30) Mougenot C., Salomir R., Palussière J., Grenier N., Moonen C. T. W., Automatic spatial and temporal temperature control for MR-guided focused ultrasound using fast 3D MR thermometry and multispiral trajectory of the focal point. Magn Reson Med 2004;52:1005-1015.

(31) Chavez S., Xiang Q. S., An L., Understanding phase maps in MRI: a new cutline phase unwrapping method. IEEE Trans Med Imaging. 2002 Aug;21(8):966-77.

(32) Conturo thE., Smith G. D., Signal to Noise in Phase Angle Reconstruction : Dynamic Range Extension Using Phase Reference Offsets, Magnetic Resonance in Medicine, 1990, 15:420-437.

(33) Moonen C.T., Spatio-temporal control of gene expression and cancer treatment using magnetic R1.3 resonance imaging-guided focused ultrasound., Clin Cancer Res. 2007 Jun 15;13(12):3482-9. 


\section{LIST OF FIGURES}

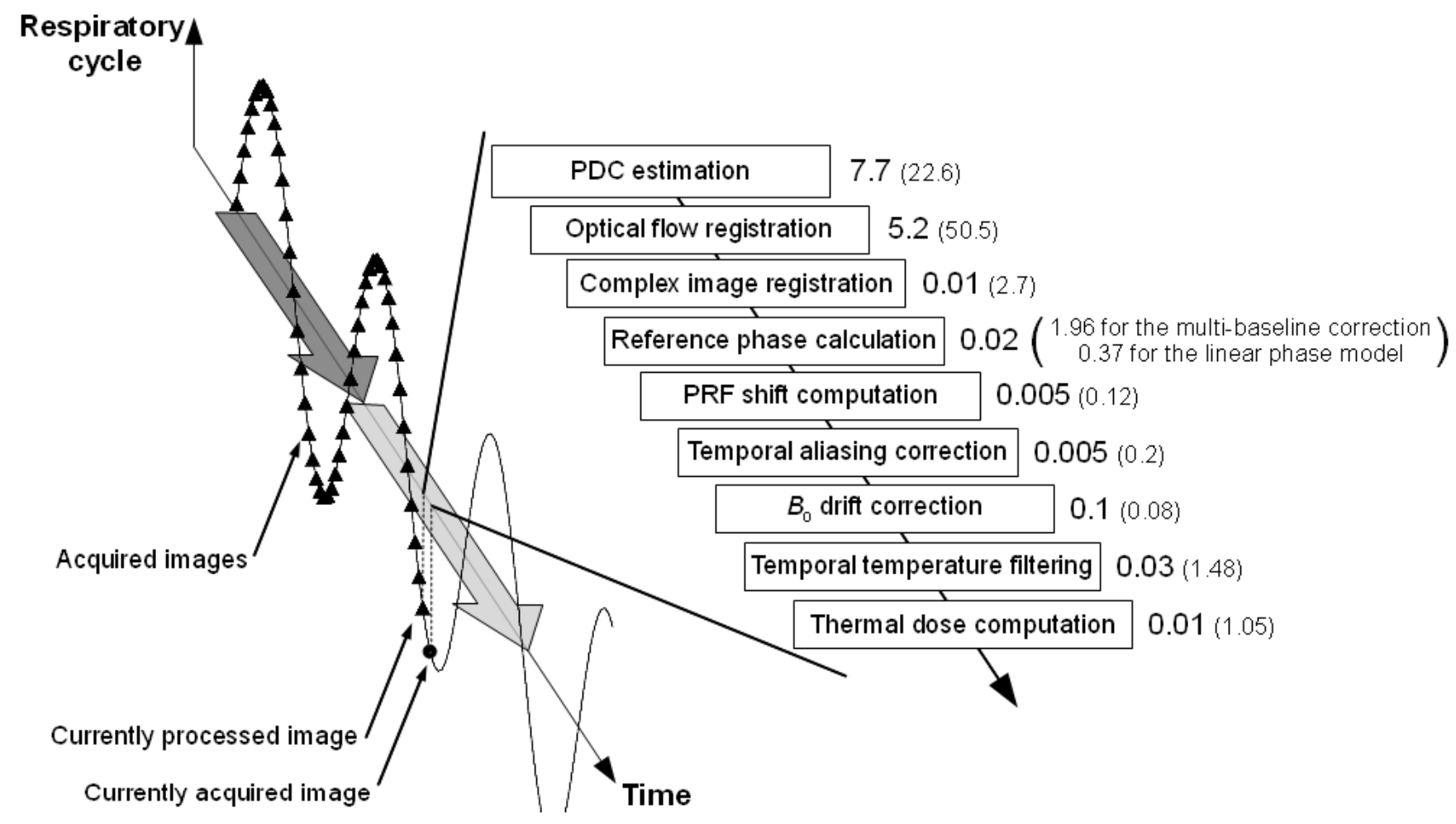

Figure 1. Data processing sequence designed for both corrections, multi-baseline and linear phase model corrections. Common to both approaches is the separation of the intervention into a pre-treatment step (dark grey arrow) and a hyperthermia step (light grey arrow). Computation times for a single image (resolution $128 \times 128$ ) are reported in milliseconds for each processing step with the CPU/GPU implementation (bold) and CPU only implementation (brackets). The most time consuming task, the image registration, was accelerated by a factor of 3 for the PDC estimation and a factor of 10 for the optical flow computation using the CPU/GPU implementation. All others tasks, being in the range of a millisecond, have been substantially reduced below $0.1 \mathrm{~ms}$. 


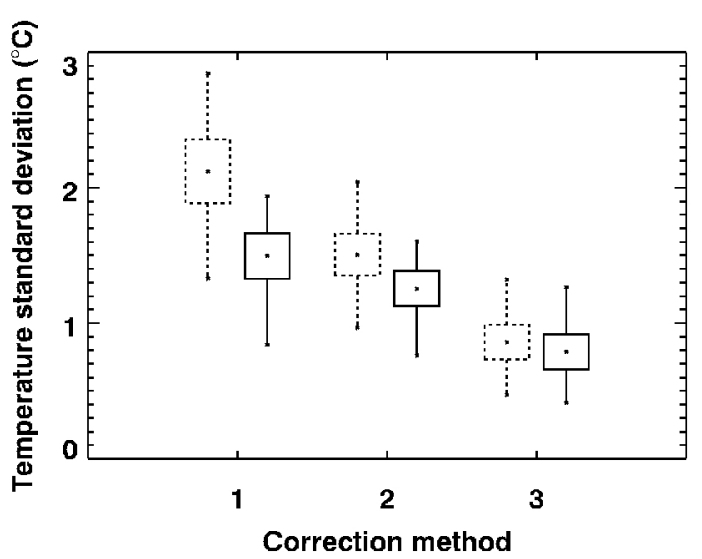

a

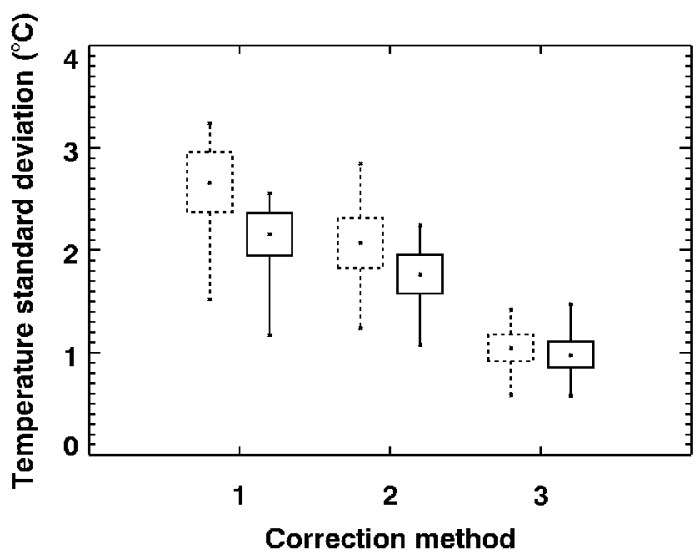

b

Figure 2. Box-and-Whisker plot of the group analysis of the temperature precision over the 11 volunteers obtained in the kidney (a) and the liver (b), with the multi-baseline approach (dash line) and with linear phase modelling approach (solid line). Plotted values correspond to the minimum (lowest point), the average (cross), the maximum (highest point) and the standard deviation (box height) values across the group, before drift correction (1), after drift correction (2) and after temporal filtering (3).

Figure 3. Temper-
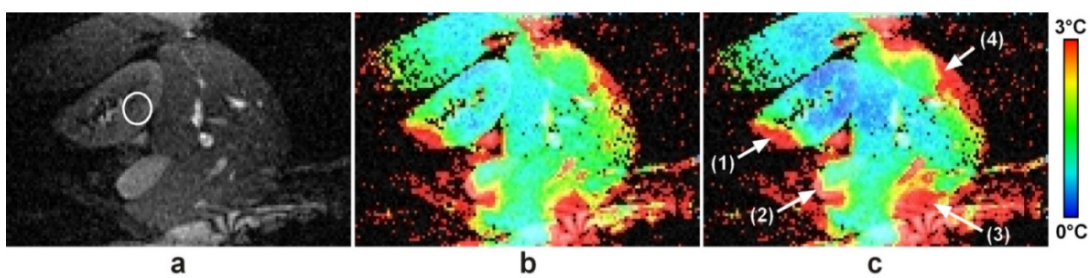

ature stability obtained in the abdomen of a healthy volunteer with each proposed correction method: (a) anatomical image depicting the ROI used for drift correction, (b) the temperature standard deviation map obtained with the multi-baseline method, (c) the temperature standard deviation map corrected with the linear phase model. White arrows indicate regions where large susceptibility variations render the temperature correction difficult: vicinity of the digestive tubes (1), vicinity of of the quadratus lumborum muscle (2), vicinity of the vertebral column (3) and upper part of the liver (4).

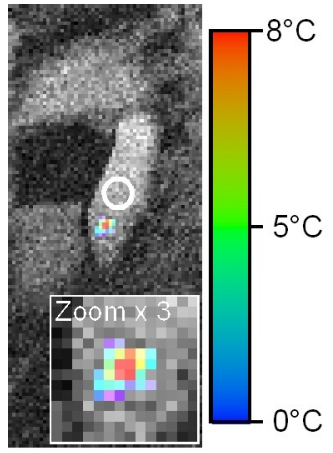

a

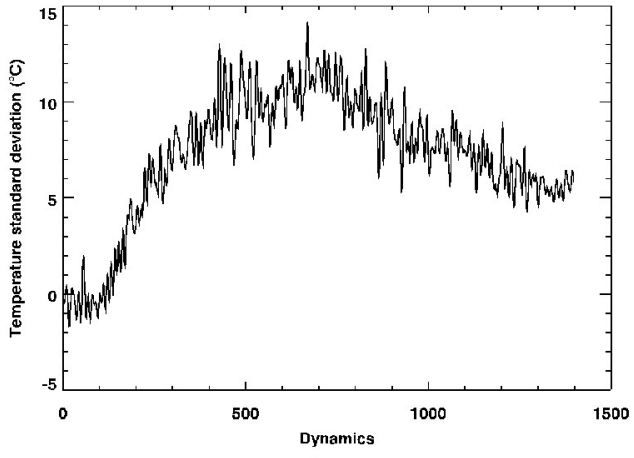

b

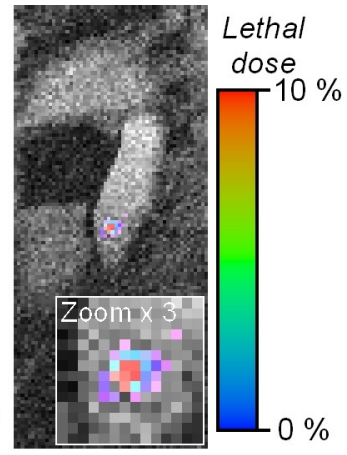

C 
Figure 4. MR-Thermometry results obtained on a pig liver during HIFU heating, corrected using the R1.7 linear phase model: (a) the temperature distribution obtained after 39 seconds of heating and the ROI used for drift correction overlayed on the anatomical image, (b) the temporal evolution of the temperatR2.8 ure at the focal point position (note that the baseline precision of $0.65{ }^{\circ} \mathrm{C}$ decreases during hyperthermia due to a change of the $T_{1}$ relaxation [33]), (c) the thermal dose map obtained at the end of the experiment. 